Marilyn Thomas and

Sally-Ann Aubrey-Smith

\section{Office for National Statistics}

\title{
The impact of Labour Force Survey and Annual Population Survey reweighting
}

\section{SUMMARY}

Reweighted Labour Force Survey (LFS) and Annual Population Survey (APS) microdata were published by the Office for National Statistics (ONS) in May 2008. The underlying weighting methodology and the statistical tool used for weighting the LFS were also changed for the reweighting programme and for subsequent LFS weighting (although not for the APS, as this already used the new methodology and tool).

This article outlines the background to the LFS and APS reweighting programme, briefly describes the changes to the weighting tool and methodology in the LFS, and presents some results of the impact of reweighting on population estimates at unitary authority/local authority district level, using APS microdata.
( n 14 May 2008, reweighted Labour Force Survey (LFS) and Annual Population Survey (APS) microdata were published by the Office for National Statistics (ONS). The reweighted LFS and APS microdata are weighted to population estimates which are based on mid-year estimates up to 2006, in line with the revised 2001 Census data released in late 2003, and the projections from the midyear estimates. The datasets they replaced had been weighted to population estimates which were based on mid-year estimates up to 2003, in line with 2001 Census data released in early 2003, and the projections from the mid-year estimates. Analysis of the reweighted microdata produces aggregates at the UK level which, by 2007 , are approximately 800,000 above the estimates produced from the unreweighted microdata for the $16+$ population $(900,000$ for the total population). However, due to the interim reweighting which ONS carries out to adjust aggregate LFS data to the latest population estimates prior to publication, the reweighting has not resulted in any change to the published aggregates. It is only when LFS and APS microdata are used to generate aggregates (which have not been interim reweighted), that the changes in population estimates due to reweighting become apparent.

When analysis is conducted at lower levels of geography, for example, unitary authority (UA)/local authority district (LAD) level, the largest changes, as may be expected, can be seen for estimates for UA/LADs that experienced the largest revisions to their population totals with reweighting. The differences are greatest for estimates of levels, that is, the actual number of people in a particular subpopulation. There has been less of an impact on rates and proportions (as revisions to population estimates produced changes in the numerator and denominator for these calculations). Differences may also be larger where the underlying base numbers in a particular subgroup are small, reflecting the larger sampling error for such estimates. Another factor to be aware of is that the underlying weighting methodology and the statistical tool used for weighting the LFS were changed for the reweighting programme and subsequent LFS weighting (but not the APS, as the APS already used the new methodology and tool). The changes to the LFS weighting methodology and tool are outlined in Box 1.

\section{Use of APS microdata}

The APS is a continuous survey of households in the United Kingdom, which is produced quarterly and contains annual data. Each dataset (known as a quarterly rolling annual dataset) consists of wave one and wave five of the quarterly LFS, and additional boost cases in England, Wales and Scotland, which are added to ensure that a minimum of 875 interviews are conducted with economically active people in each local education authority. Each APS dataset contains approximately 170,000 households and 360,000 individuals. The primary purpose of the APS is to provide estimates for labour market and socio- 


\section{Box 1 \\ Methodological improvements to LFS weighting}

The methodology for weighting LFS data was changed for the reweighting exercise from raking ratio estimation to calibration weighting within a generalised regression (GREG) framework, using the Statistics Canada Generalised Estimation System. The raking ratio method of weighting entailed using multistage iterative weighting in which the design-weighted sample was raked to known population totals. Each stage of the procedure corrected for a different cause of LFS non-response. Stage one corrected for non-response at local authority level. Stage two corrected for non-response by age-group and sex. Stage three corrected for non-response by region, age group and sex. The weights were deemed to have converged according to any of three separate criteria. The first was that the weighted sample met the set constraints within a specified tolerance. The second was that consecutive iterations did not differ by more than a given tolerance. The third was that a preset number of iterations had been completed. The advantage of the old methodology was that weights were always positive when given positive constraints, but the disadvantages were that the methodology was not supported by mature theory, although convergence appeared to be practically assured. Also, definitive variance estimates could not be produced (although assumptions were made in order to produce variance estimates), and there was no control over the variance within the assumed model. Full details of the three-stage iterative weighting procedure can be found in the LFS User Guide Volume 1, Section 10. ${ }^{1}$

The new LFS weighting tool, and the methodology it uses, carry out calibration weighting in a single process to the same population groups as the old weighting, and are both more efficient and statistically robust. The tool also facilitates the identification and correction of data quality issues. The new weighting methodology has a number of advantages over the old one in terms of a well-developed theoretical base and the capacity to produce good variance estimates, and parameters within GREG allow for modelling of the variance within the assumed model. The new methodology was not expected to result in much change to LFS estimates at higher levels of geography, due to the large sample size, although it was expected that there were likely to be some changes within small domains, for example, when examining gender or ethnicity at UA/LAD level. However, while the LFS provides robust estimates at UK level, the APS, which also uses the GREG framework, is the preferred source for analysis at subnational level. economic analyses at subnational level, and the APS is the recommended source of statistical information for analysis at $\mathrm{UA} / \mathrm{LAD}$ level. Some analysis has been conducted of the change in population estimates at UA/LAD level as a result of reweighting the APS, using January to December 2006 APS microdata. The changes observed are reported below.

\section{Change in APS population estimates following reweighting}

The data reported have been generated from a comparison of estimates by UA/ LAD using un-reweighted APS data and reweighted APS data, using January to December 2006 APS microdata. Data are provided on the percentage change in the estimates for people aged $16+$ in the population and in employment for the total $16+$ population and the UK born and non-UK born population subgroups (note that non-UK born include those from the Republic of Ireland, and that Northern Ireland is included as a single UA/LAD in the analysis). Most of the data included in the analysis meet the ONS criteria for statistical robustness, as outlined in

Box 2. The exceptions to this rule are for estimates of non-UK born population and employment in some UA/LADs, due to the small sample sizes of this subgroup at this level of analysis.

Figure 1 shows the percentage change in the population aged $16+$ at UA/LAD level as a result of reweighting, for the total population and for UK born and non-UK born population subgroups. While the changes due to reweighting range between +22 and -6 per cent, most of the UA/LADs experienced a change within the range +5 to -5 per cent for the total population and for the UK born and non-UK born subgroups.

Figure 2 shows the banded percentage change in the $16+$ population at UA/LAD level for the total population. As a result of reweighting, the majority (68.2 per cent) of UA/LADs experienced an increase in the $16+$ population of between 0 and 5 per cent, a further 5.5 per cent experienced an increase of between 5 and 10 per cent, and 24.4 per cent experienced a decrease of between 0 and 5 per cent.
Figure 3 shows the banded percentage change in the $16+$ population at UA/LAD level for the UK born population. The results are similar to those produced for the total population, with 67.2 per cent of UA/ LADs experiencing an increase of between 0 and 5 per cent, a further 5.5 per cent experiencing an increase of between 5 and 10 per cent, and 25.9 per cent of UA/LADs experiencing a decrease of between 0 and 5 per cent.

Figure 4 shows the banded percentage change in the $16+$ population at UA/LAD level for the non-UK born population. The percentage of UA/LADs experiencing an increase of between 0 and 5 per cent for the non-UK born $16+$ population $(61.7$ per cent) was lower than for the UK born population, but was still the most common

\section{Box 2}

\section{Sampling variability}

As is the case with all sample surveys, estimates from the APS are subject to sampling variability. The larger the population whose size is being estimated, the more precise the estimate is (that is, the higher the quality), and vice versa. One estimate of the variability of the estimates from all possible samples is the coefficient of variation (CV). The CV provides a measure of dispersion, and a higher CV indicates greater dispersion (or margin of error). As the CV increases, the estimate itself becomes less reliable, and vice-versa. ONS publishes and categorises all APS estimates according to their statistical robustness, which are shown below.

$0 \leq \mathrm{CV}<5 \quad$ Estimates are considered precise

$5 \leq \mathrm{CV}<10 \quad$ Estimates are considered reasonably precise

$10 \leq \mathrm{CV}<20 \quad$ Estimates are considered acceptable

CV $\geq 20 \quad$ Estimates are considered too unreliable for practical purposes 


\section{Figure 1}

\section{Percentage change in 16+ population due to reweighting: by unitary} authority/local authority district



\section{Figure 2}

\section{Percentage change in total $16+$ population due to reweighting}

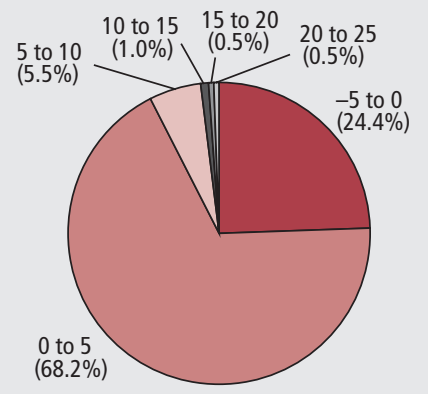

Figure 3

Percentage change in UK born $16+$ population due to reweighting

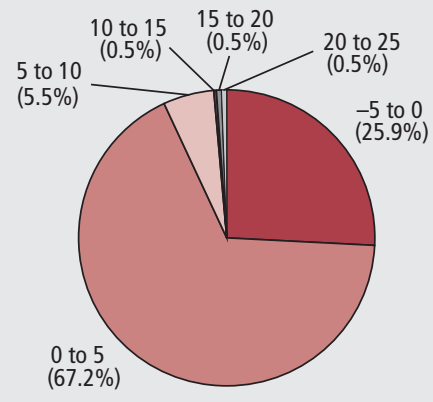

Figure 4

Percentage change in non-UK born $16+$ population due to reweighting

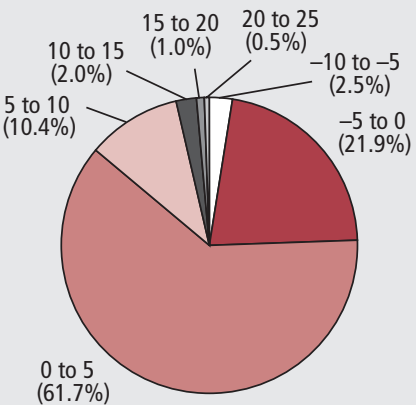

change. Consequently, the percentage of UA/LADs with an increase of between 5 and 10 per cent was higher (10.4 per cent), while 21.9 per cent experienced a decrease of between 0 and 5 per cent, as a result of reweighting.

Figure 5 shows the percentage change in employment levels at UA/LAD level as a result of reweighting, for the total population and for UK born and non-UK born subgroups. While the range of the percentage change in employment levels varies between +23 and -6 per cent, the majority of UA/LADs experienced a change of between +5 and -5 per cent.

Figure 6 shows the banded percentage change in employment levels at UA/LAD level for the total population. While 62.7 per cent of UA/LADs experienced an increase of between 0 and 5 per cent, a further 10.4 per cent experienced an increase of between 5 and 10 per cent, and 21.9 per cent experienced a decrease of between 0 and 5 per cent, as a result of reweighting.

Figure 7 shows the banded percentage change in employment levels at UA/LAD level for the UK born population. The data are very similar to that for the total population, with 62.7 per cent of UA/LADs experiencing an increase of between 0 and 5 per cent, a further 8.5 per cent experiencing an increase of between 5 and 10 per cent, and 22.4 per cent experiencing a decrease of between 0 and -5 per cent.

Figure 8 shows the banded percentage change in employment levels at UA/LAD level for the non-UK born population. Reweighting resulted in an increase in employment levels of between 0 and 5 per cent for 52.2 per cent of UA/LADs, an increase of between 5 and 10 per cent for a further 14.4 per cent, and a reduction of between 0 and 5 per cent for 24.9 per cent of UA/LADs.

\section{Conclusion}

The LFS and APS reweighting programme that was conducted between 2007 and early 2008 culminated in the release by ONS of reweighted LFS and APS microdata on 14 May 2008, bringing LFS microdata in line with published LFS aggregates. This article has outlined the background to the reweighting programme, the changes to the weighting methodology and tool used for LFS weighting, and the impact of reweighting on population estimates at UA/LAD level using APS microdata. Analysis of APS microdata, which is the recommended source for estimates at $\mathrm{UA} / \mathrm{LAD}$ level, revealed that the majority of UA/LADs experienced a change of 


\section{Figure 5}

Percentage change in employment levels due to reweighting: by unitary authority/local authority district

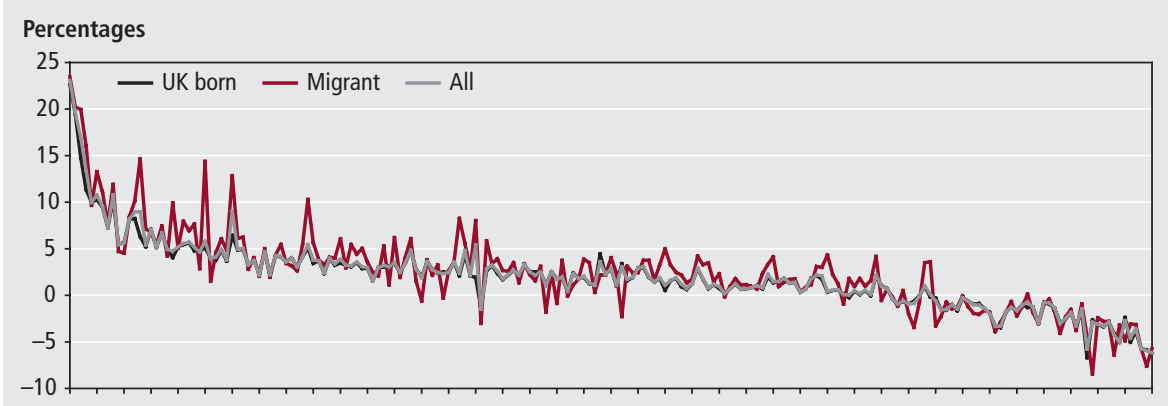

Figure 6

Percentage change in total population employment levels due to reweighting

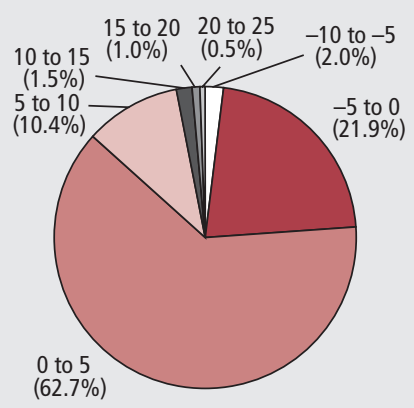

Figure 7

Percentage change in UK born population employment levels due to reweighting

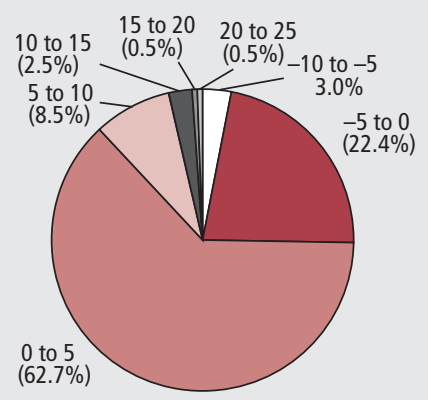

Figure 8

Percentage change in non-UK born population employment levels due to reweighting

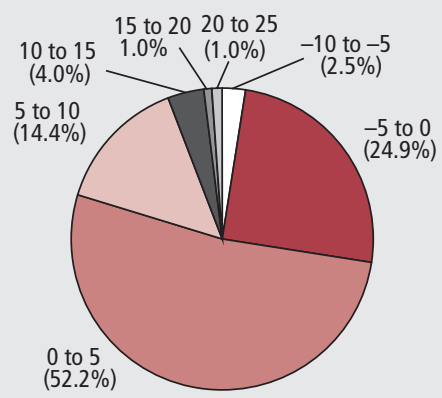

between +5 and -5 per cent in the $16+$ population and employment levels, for the total population and for the UK born and non-UK born population subgroups. ONS is currently planning the next LFS and APS reweighting programme, and full details will be announced when the plans have been finalised.

\section{Notes}

1 See www.statistics.gov.uk/statbase/ product.asp?vlnk=1537

\section{CONTACT}

(《)elmr@ons.gsi.gov.uk 
Annual Population Survey, UK 16+ population and employment levels: by unitary authority/local authority district, January to December 2006

Numbers and percentages

\begin{tabular}{|c|c|c|c|c|c|c|c|c|}
\hline \multirow[b]{2}{*}{ Local authority } & \multicolumn{3}{|c|}{ Unreweighted (PWT03) } & \multicolumn{3}{|c|}{ Reweighted (PWT07) } & \multirow{2}{*}{$\begin{array}{r}\text { Difference in } \\
16+ \\
\text { population } \\
\text { (per cent) }\end{array}$} & \multirow{2}{*}{$\begin{array}{r}\text { Difference in } \\
\text { employment } \\
\text { levels } \\
\text { (per cent) }\end{array}$} \\
\hline & $16+$ population & $\begin{array}{r}\text { Employment } \\
\text { levels }\end{array}$ & $\begin{array}{r}\text { Coefficient of } \\
\text { variation }\end{array}$ & $16+$ population & $\begin{array}{r}\text { Employment } \\
\text { levels }\end{array}$ & $\begin{array}{r}\text { Coefficient of } \\
\text { variation }\end{array}$ & & \\
\hline Westminster & 171,166 & 95,495 & 7.80 & 209,257 & 117,543 & 7.77 & 22.25 & 23.09 \\
\hline Manchester & 309,115 & 169,415 & 5.46 & 362,108 & 202,708 & 5.40 & 17.14 & 19.65 \\
\hline Southwark & 190,991 & 109,901 & 8.23 & 216,925 & 128,503 & 8.11 & 13.58 & 16.93 \\
\hline Camden & 167,985 & 99,403 & 7.68 & 185,837 & 112,700 & 7.59 & 10.63 & 13.38 \\
\hline Bristol, City of UA & 313,008 & 194,371 & 5.69 & 338,956 & 213,741 & 5.65 & 8.29 & 9.97 \\
\hline Nottingham UA & 219,232 & 116,158 & 5.56 & 236,199 & 128,652 & 5.48 & 7.74 & 10.76 \\
\hline Kingston Upon Hull, City of UA & 193,103 & 101,882 & 5.87 & 206,292 & 111,643 & 5.80 & 6.83 & 9.58 \\
\hline Middlesbrough UA & 101,978 & 55,083 & 5.52 & 108,644 & 59,104 & 5.50 & 6.54 & 7.30 \\
\hline Lambeth & 205,478 & 115,776 & 8.41 & 218,025 & 128,280 & 8.23 & 6.11 & 10.80 \\
\hline North Somerset UA & 152,993 & 96,353 & 5.28 & 161,954 & 101,601 & 5.29 & 5.86 & 5.45 \\
\hline Stockton-on-Tees UA & 139,882 & 82,964 & 5.73 & 147,649 & 87,641 & 5.72 & 5.55 & 5.64 \\
\hline Southampton UA & 178,477 & 104,104 & 5.74 & 188,202 & 112,660 & 5.67 & 5.45 & 8.22 \\
\hline Wandsworth & 222,116 & 137,777 & 8.42 & 234,126 & 150,088 & 8.28 & 5.41 & 8.94 \\
\hline Islington & 143,101 & 84,440 & 7.90 & 150,631 & 92,026 & 7.76 & 5.26 & 8.98 \\
\hline Newcastle Upon Tyne & 205,877 & 110,654 & 5.49 & 216,645 & 116,659 & 5.48 & 5.23 & 5.43 \\
\hline York UA & 151,599 & 95,170 & 5.49 & 159,169 & 101,893 & 5.43 & 4.99 & 7.06 \\
\hline Derby UA & 179,653 & 101,585 & 5.43 & 188,589 & 106,841 & 5.42 & 4.97 & 5.17 \\
\hline North East Lincolnshire UA & 119,358 & 67,924 & 5.71 & 124,922 & 72,485 & 5.65 & 4.66 & 6.71 \\
\hline Halton UA & 89,618 & 50,285 & 5.74 & 93,759 & 52,762 & 5.73 & 4.62 & 4.93 \\
\hline Edinburgh, City of & 370,529 & 231,725 & 5.41 & 387,317 & 242,747 & 5.41 & 4.53 & 4.76 \\
\hline Eilean Siar, Orkney \& Shetland & 52,334 & 33,607 & 6.34 & 54,704 & 35,354 & 6.32 & 4.53 & 5.20 \\
\hline Highland & 164,882 & 103,311 & 6.10 & 172,315 & 109,054 & 6.07 & 4.51 & 5.56 \\
\hline North Lincolnshire UA & 121,018 & 71,345 & 5.81 & 126,456 & 75,433 & 5.78 & 4.49 & 5.73 \\
\hline Leeds & 578,830 & 368,652 & 4.52 & 604,732 & 387,638 & 4.51 & 4.47 & 5.15 \\
\hline Newport & 105,371 & 61,013 & 5.36 & 109,775 & 63,856 & 5.35 & 4.18 & 4.66 \\
\hline Aberdeen City & 164,822 & 108,760 & 5.52 & 171,281 & 115,060 & 5.48 & 3.92 & 5.79 \\
\hline Knowsley & 114,203 & 61,661 & 5.29 & 118,622 & 64,085 & 5.29 & 3.87 & 3.93 \\
\hline Hounslow & 165,768 & 106,174 & 7.38 & 172,136 & 110,546 & 7.37 & 3.84 & 4.12 \\
\hline Salford & 167,862 & 96,405 & 5.08 & 174,241 & 101,146 & 5.05 & 3.80 & 4.92 \\
\hline Bradford & 367,946 & 208,060 & 5.74 & 381,433 & 215,996 & 5.74 & 3.67 & 3.81 \\
\hline Tower Hamlets & 162,241 & 74,707 & 8.58 & 168,182 & 81,477 & 8.36 & 3.66 & 9.06 \\
\hline Perth and Kinross & 108,147 & 65,319 & 4.94 & 112,107 & 68,545 & 4.91 & 3.66 & 4.94 \\
\hline Cardiff & 245,789 & 138,148 & 5.51 & 254,699 & 145,117 & 5.47 & 3.63 & 5.04 \\
\hline Gwynedd & 91,725 & 51,618 & 5.36 & 94,954 & 53,307 & 5.37 & 3.52 & 3.27 \\
\hline Merthyr Tydfil & 42,751 & 21,042 & 7.71 & 44,229 & 21,830 & 7.69 & 3.46 & 3.74 \\
\hline Kensington and Chelsea & 144,747 & 82,194 & 8.24 & 149,709 & 84,003 & 8.29 & 3.43 & 2.20 \\
\hline Plymouth UA & 191,484 & 111,021 & 5.76 & 198,017 & 116,228 & 5.73 & 3.41 & 4.69 \\
\hline Anglesey, Isle of & 53,682 & 28,418 & 6.15 & 55,484 & 29,048 & 6.18 & 3.36 & 2.22 \\
\hline Brent & 205,057 & 116,327 & 8.98 & 211,891 & 121,297 & 8.94 & 3.33 & 4.27 \\
\hline North Tyneside & 155,110 & 89,998 & 5.50 & 160,210 & 93,747 & 5.48 & 3.29 & 4.17 \\
\hline Bridgend & 102,184 & 55,764 & 5.48 & 105,542 & 57,768 & 5.47 & 3.29 & 3.59 \\
\hline Neath Port Talbot & 106,880 & 53,773 & 5.39 & 110,392 & 55,914 & 5.37 & 3.29 & 3.98 \\
\hline Carmarthenshire & 138,372 & 73,220 & 5.38 & 142,871 & 75,447 & 5.38 & 3.25 & 3.04 \\
\hline Rhondda, Cynon, Taff & 179,950 & 96,786 & 5.55 & 185,742 & 100,817 & 5.52 & 3.22 & 4.16 \\
\hline Dundee City & 112,994 & 62,356 & 5.28 & 116,627 & 65,752 & 5.22 & 3.22 & 5.45 \\
\hline Sandwell & 215,011 & 115,186 & 5.71 & 221,902 & 119,540 & 5.69 & 3.20 & 3.78 \\
\hline Durham & 389,259 & 220,538 & 5.42 & 401,475 & 228,701 & 5.40 & 3.14 & 3.70 \\
\hline Glasgow City & 461,816 & 247,528 & 5.47 & 476,123 & 253,440 & 5.49 & 3.10 & 2.39 \\
\hline Northamptonshire & 507,586 & 335,831 & 4.74 & 523,134 & 349,217 & 4.72 & 3.06 & 3.99 \\
\hline Birmingham & 749,977 & 391,955 & 4.61 & 772,947 & 405,252 & 4.60 & 3.06 & 3.39 \\
\hline
\end{tabular}


Table A1 continued

Numbers and percentages

\begin{tabular}{|c|c|c|c|c|c|c|c|c|}
\hline \multirow[b]{2}{*}{ Local authority } & \multicolumn{3}{|c|}{ Unreweighted (PWT03) } & \multicolumn{3}{|c|}{ Reweighted (PWT07) } & \multirow{2}{*}{$\begin{array}{r}\text { Difference in } \\
16+ \\
\text { population } \\
\text { (per cent) }\end{array}$} & \multirow{2}{*}{$\begin{array}{r}\text { Difference in } \\
\text { employment } \\
\text { levels } \\
\text { (per cent) }\end{array}$} \\
\hline & $16+$ population & $\begin{array}{r}\text { Employment } \\
\text { levels } \\
\end{array}$ & $\begin{array}{r}\text { Coefficient of } \\
\text { variation } \\
\end{array}$ & $16+$ population & $\begin{array}{r}\text { Employment } \\
\text { levels } \\
\end{array}$ & $\begin{array}{r}\text { Coefficient of } \\
\text { variation }\end{array}$ & & \\
\hline Peterborough UA & 121,918 & 76,306 & 5.48 & 125,584 & 79,270 & 5.46 & 3.01 & 3.88 \\
\hline Hartlepool UA & 70,399 & 36,279 & 5.69 & 72,511 & 37,452 & 5.68 & 3.00 & 3.23 \\
\hline Calderdale & 150,520 & 91,731 & 5.70 & 154,974 & 94,647 & 5.70 & 2.96 & 3.18 \\
\hline Portsmouth UA & 150,729 & 96,314 & 5.67 & 155,167 & 99,762 & 5.65 & 2.94 & 3.58 \\
\hline Sheffield & 409,092 & 223,909 & 5.61 & 421,042 & 230,837 & 5.60 & 2.92 & 3.09 \\
\hline Bath and North East Somerset UA & 139,181 & 82,710 & 5.67 & 143,147 & 85,152 & 5.66 & 2.85 & 2.95 \\
\hline Powys & 102,739 & 61,313 & 5.43 & 105,663 & 62,279 & 5.46 & 2.85 & 1.58 \\
\hline Barnsley & 174,026 & 94,473 & 5.59 & 178,971 & 97,277 & 5.58 & 2.84 & 2.97 \\
\hline Suffolk & 542,946 & 326,442 & 4.77 & 558,056 & 336,919 & 4.76 & 2.78 & 3.21 \\
\hline Aberdeenshire & 181,805 & 120,831 & 5.22 & 186,798 & 124,564 & 5.21 & 2.75 & 3.09 \\
\hline Nottinghamshire & 598,209 & 357,875 & 4.51 & 614,624 & 370,447 & 4.49 & 2.74 & 3.51 \\
\hline St. Helens & 139,402 & 77,081 & 5.26 & 143,191 & 78,985 & 5.27 & 2.72 & 2.47 \\
\hline Sunderland & 220,626 & 119,646 & 5.73 & 226,531 & 123,819 & 5.70 & 2.68 & 3.49 \\
\hline Clackmannanshire & 37,637 & 21,016 & 9.17 & 38,610 & 22,034 & 9.07 & 2.59 & 4.84 \\
\hline Blaenau Gwent & 53,848 & 27,115 & 6.91 & 55,215 & 27,871 & 6.90 & 2.54 & 2.79 \\
\hline Angus & 85,273 & 53,197 & 4.91 & 87,417 & 54,192 & 4.93 & 2.51 & 1.87 \\
\hline Isle of Wight UA & 104,827 & 58,187 & 5.45 & 107,441 & 60,296 & 5.42 & 2.49 & 3.62 \\
\hline Liverpool & 337,432 & 175,921 & 5.48 & 345,826 & 180,733 & 5.47 & 2.49 & 2.74 \\
\hline Rotherham & 198,144 & 112,297 & 5.48 & 202,930 & 115,054 & 5.48 & 2.42 & 2.46 \\
\hline Darlington UA & 77,670 & 46,074 & 5.51 & 79,456 & 47,153 & 5.51 & 2.30 & 2.34 \\
\hline East Riding of Yorkshire UA & 259,338 & 155,053 & 5.22 & 265,206 & 158,836 & 5.21 & 2.26 & 2.44 \\
\hline Leicester UA & 220,145 & 127,046 & 5.18 & 225,051 & 131,605 & 5.14 & 2.23 & 3.59 \\
\hline Wakefield & 252,054 & 151,718 & 5.76 & 257,608 & 155,325 & 5.75 & 2.20 & 2.38 \\
\hline Waltham Forest & 171,007 & 97,219 & 8.35 & 174,775 & 101,899 & 8.24 & 2.20 & 4.81 \\
\hline Walsall & 197,878 & 102,316 & 5.96 & 202,159 & 104,483 & 5.96 & 2.16 & 2.12 \\
\hline Newham & 188,272 & 93,685 & 8.07 & 192,243 & 98,750 & 7.95 & 2.11 & 5.41 \\
\hline Rutland UA & 30,014 & 18,729 & 9.08 & 30,627 & 18,453 & 9.24 & 2.04 & -1.47 \\
\hline Swansea & 180,595 & 97,429 & 5.49 & 184,211 & 100,195 & 5.47 & 2.00 & 2.84 \\
\hline Greenwich & 169,997 & 99,829 & 7.95 & 173,374 & 103,071 & 7.90 & 1.99 & 3.25 \\
\hline Coventry & 240,767 & 141,455 & 5.47 & 245,500 & 145,148 & 5.45 & 1.97 & 2.61 \\
\hline Havering & 181,390 & 108,171 & 7.92 & 184,925 & 110,089 & 7.93 & 1.95 & 1.77 \\
\hline Milton Keynes UA & 166,305 & 115,669 & 5.91 & 169,541 & 118,248 & 5.90 & 1.95 & 2.23 \\
\hline Cornwall and The Isles of Scilly & 421,281 & 235,966 & 5.57 & 429,450 & 242,349 & 5.55 & 1.94 & 2.71 \\
\hline Lancashire & 910,438 & 532,665 & 3.81 & 928,013 & 544,142 & 3.81 & 1.93 & 2.15 \\
\hline Lewisham & 201,424 & 124,122 & 7.77 & 205,281 & 128,231 & 7.71 & 1.91 & 3.31 \\
\hline Kent & $1,072,446$ & 637,456 & 3.48 & $1,092,946$ & 653,552 & 3.47 & 1.91 & 2.53 \\
\hline Merton & 156,406 & 100,443 & 7.25 & 159,394 & 102,611 & 7.24 & 1.91 & 2.16 \\
\hline Devon & 585,663 & 343,177 & 4.95 & 596,779 & 351,811 & 4.94 & 1.90 & 2.52 \\
\hline West Dunbartonshire & 72,441 & 42,729 & 5.14 & 73,792 & 43,162 & 5.16 & 1.86 & 1.01 \\
\hline Blackburn with Darwen UA & 102,521 & 57,117 & 5.24 & 104,429 & 58,559 & 5.22 & 1.86 & 2.52 \\
\hline South Ayrshire & 89,648 & 50,173 & 5.32 & 91,291 & 51,023 & 5.33 & 1.83 & 1.69 \\
\hline Norfolk & 664,031 & 387,677 & 4.47 & 676,108 & 395,308 & 4.46 & 1.82 & 1.97 \\
\hline Wirral & 244,005 & 132,739 & 6.02 & 248,202 & 133,296 & 6.06 & 1.72 & 0.42 \\
\hline Leicestershire & 505,369 & 327,154 & 4.78 & 514,043 & 334,754 & 4.76 & 1.72 & 2.32 \\
\hline Redbridge & 193,555 & 110,572 & 8.48 & 196,867 & 112,569 & 8.48 & 1.71 & 1.81 \\
\hline Doncaster & 228,434 & 124,435 & 5.71 & 232,313 & 126,959 & 5.70 & 1.70 & 2.03 \\
\hline Wigan & 240,070 & 140,064 & 6.19 & 244,078 & 141,938 & 6.20 & 1.67 & 1.34 \\
\hline South Lanarkshire & 242,356 & 150,203 & 5.39 & 246,397 & 151,814 & 5.40 & 1.67 & 1.07 \\
\hline Haringey & 178,830 & 106,660 & 7.66 & 181,787 & 110,517 & 7.59 & 1.65 & 3.62 \\
\hline Essex & $1,070,822$ & 650,999 & 3.58 & $1,088,049$ & 665,667 & 3.57 & 1.61 & 2.25 \\
\hline Falkirk & 117,631 & 72,372 & 5.30 & 119,522 & 74,456 & 5.27 & 1.61 & 2.88 \\
\hline Torfaen & 71,051 & 38,860 & 5.40 & 72,186 & 39,275 & 5.41 & 1.60 & 1.07 \\
\hline Moray & 68,214 & 41,883 & 4.73 & 69,230 & 43,169 & 4.69 & 1.49 & 3.07 \\
\hline Bedfordshire & 314,234 & 198,910 & 6.23 & 318,902 & 202,349 & 6.23 & 1.49 & 1.73 \\
\hline Redcar and Cleveland UA & 109,732 & 59,851 & 5.50 & 111,358 & 61,009 & 5.49 & 1.48 & 1.93 \\
\hline
\end{tabular}




\begin{tabular}{|c|c|c|c|c|c|c|c|c|}
\hline \multirow[b]{2}{*}{ Local authority } & \multicolumn{3}{|c|}{ Unreweighted (PWT03) } & \multicolumn{3}{|c|}{ Reweighted (PWT07) } & \multirow{2}{*}{$\begin{array}{r}\text { Difference in } \\
16+ \\
\text { population } \\
\text { (per cent) }\end{array}$} & \multirow{2}{*}{$\begin{array}{r}\text { Difference in } \\
\text { employment } \\
\text { levels } \\
\text { (per cent) }\end{array}$} \\
\hline & $16+$ population & $\begin{array}{r}\text { Employment } \\
\text { levels }\end{array}$ & $\begin{array}{r}\text { Coefficient of } \\
\text { variation }\end{array}$ & $16+$ population & $\begin{array}{r}\text { Employment } \\
\text { levels }\end{array}$ & $\begin{array}{r}\text { Coefficient of } \\
\text { variation }\end{array}$ & & \\
\hline Lincolnshire & 544,524 & 315,647 & 4.96 & 552,540 & 324,785 & 4.92 & 1.47 & 2.90 \\
\hline Wolverhampton & 185,404 & 99,015 & 5.87 & 188,084 & 102,082 & 5.82 & 1.45 & 3.10 \\
\hline Renfrewshire & 134,609 & 82,213 & 5.29 & 136,542 & 83,855 & 5.28 & 1.44 & 2.00 \\
\hline Solihull & 160,601 & 98,698 & 5.55 & 162,900 & 100,116 & 5.55 & 1.43 & 1.44 \\
\hline North Yorkshire & 462,901 & 278,519 & 5.33 & 469,292 & 283,806 & 5.31 & 1.38 & 1.90 \\
\hline Oxfordshire & 488,599 & 329,086 & 4.86 & 495,179 & 332,657 & 4.87 & 1.35 & 1.09 \\
\hline Staffordshire & 650,012 & 397,985 & 4.68 & 658,334 & 404,288 & 4.68 & 1.28 & 1.58 \\
\hline Stoke-on-Trent UA & 189,617 & 104,876 & 5.61 & 192,032 & 106,845 & 5.59 & 1.27 & 1.88 \\
\hline Kingston upon Thames & 123,382 & 80,955 & 7.46 & 124,924 & 81,944 & 7.46 & 1.25 & 1.22 \\
\hline Sutton & 143,011 & 94,935 & 8.30 & 144,795 & 95,635 & 8.32 & 1.25 & 0.74 \\
\hline Cumbria & 395,791 & 232,300 & 5.73 & 400,631 & 235,249 & 5.73 & 1.22 & 1.27 \\
\hline Dumfries and Galloway & 118,916 & 69,940 & 5.34 & 120,275 & 71,991 & 5.30 & 1.14 & 2.93 \\
\hline South Tyneside & 119,735 & 61,181 & 5.73 & 121,092 & 62,281 & 5.71 & 1.13 & 1.80 \\
\hline Gateshead & 152,762 & 85,798 & 5.34 & 154,424 & 86,463 & 5.35 & 1.09 & 0.78 \\
\hline Kirklees & 305,765 & 185,938 & 5.56 & 309,089 & 188,128 & 5.56 & 1.09 & 1.18 \\
\hline Cambridgeshire & 466,506 & 296,730 & 5.21 & 471,260 & 299,542 & 5.21 & 1.02 & 0.95 \\
\hline Windsor and Maidenhead UA & 107,142 & 70,842 & 5.18 & 108,208 & 70,973 & 5.20 & 0.99 & 0.18 \\
\hline Derbyshire & 596,463 & 368,087 & 4.51 & 602,337 & 370,636 & 4.51 & 0.98 & 0.69 \\
\hline Northern Ireland & $1,324,363$ & 749,954 & 2.78 & $1,337,318$ & 759,237 & 2.77 & 0.98 & 1.24 \\
\hline Inverclyde & 65,052 & 36,959 & 5.75 & 65,678 & 37,208 & 5.76 & 0.96 & 0.67 \\
\hline Tameside & 169,234 & 101,348 & 5.34 & 170,856 & 102,055 & 5.35 & 0.96 & 0.70 \\
\hline North Ayrshire & 107,869 & 59,329 & 5.31 & 108,877 & 59,824 & 5.32 & 0.93 & 0.83 \\
\hline Shropshire & 229,395 & 134,568 & 5.80 & 231,486 & 135,950 & 5.80 & 0.91 & 1.03 \\
\hline Oldham & 165,975 & 95,833 & 5.72 & 167,461 & 96,615 & 5.72 & 0.90 & 0.82 \\
\hline Luton UA & 142,548 & 82,295 & 5.99 & 143,775 & 84,171 & 5.95 & 0.86 & 2.28 \\
\hline Somerset & 407,951 & 237,271 & 5.90 & 411,391 & 240,761 & 5.88 & 0.84 & 1.47 \\
\hline Torbay & 105,163 & 58,011 & 5.60 & 106,015 & 58,878 & 5.58 & 0.81 & 1.49 \\
\hline North Lanarkshire & 255,054 & 149,881 & 5.30 & 257,067 & 152,609 & 5.27 & 0.79 & 1.82 \\
\hline Gloucestershire & 459,179 & 288,198 & 5.14 & 462,674 & 292,069 & 5.13 & 0.76 & 1.34 \\
\hline Fife & 287,621 & 172,994 & 5.42 & 289,623 & 175,403 & 5.40 & 0.70 & 1.39 \\
\hline Sefton & 222,249 & 118,499 & 5.63 & 223,766 & 118,886 & 5.64 & 0.68 & 0.33 \\
\hline Surrey & 854,088 & 552,222 & 3.82 & 859,583 & 556,110 & 3.82 & 0.64 & 0.70 \\
\hline Swindon & 147,233 & 95,938 & 5.78 & 148,031 & 97,651 & 5.75 & 0.54 & 1.79 \\
\hline East Ayrshire & 95,232 & 53,619 & 5.44 & 95,715 & 54,784 & 5.39 & 0.51 & 2.17 \\
\hline Barking and Dagenham & 124,812 & 64,788 & 8.19 & 125,442 & 66,151 & 8.12 & 0.50 & 2.10 \\
\hline Northumberland & 250,366 & 140,915 & 5.77 & 251,625 & 141,495 & 5.78 & 0.50 & 0.41 \\
\hline Cheshire & 546,717 & 334,413 & 5.07 & 549,429 & 336,390 & 5.06 & 0.50 & 0.59 \\
\hline Warwickshire & 413,933 & 261,940 & 4.90 & 415,608 & 263,474 & 4.90 & 0.40 & 0.59 \\
\hline Blackpool UA & 110,681 & 59,278 & 5.66 & 111,075 & 59,324 & 5.67 & 0.36 & 0.08 \\
\hline Bexley & 174,155 & 107,922 & 7.61 & 174,770 & 107,955 & 7.62 & 0.35 & 0.03 \\
\hline Hertfordshire & 828,881 & 529,190 & 3.84 & 831,702 & 531,819 & 3.84 & 0.34 & 0.50 \\
\hline Dudley & 240,781 & 142,078 & 5.69 & 241,493 & 142,332 & 5.69 & 0.30 & 0.18 \\
\hline Trafford & 167,543 & 105,874 & 5.40 & 168,030 & 106,467 & 5.39 & 0.29 & 0.56 \\
\hline Rochdale & 159,316 & 94,444 & 5.58 & 159,605 & 94,534 & 5.58 & 0.18 & 0.10 \\
\hline Scot Borders, The & 88,793 & 52,877 & 5.38 & 88,908 & 54,036 & 5.33 & 0.13 & 2.19 \\
\hline Worcestershire & 443,846 & 282,141 & 5.15 & 444,254 & 284,932 & 5.13 & 0.09 & 0.99 \\
\hline Enfield & 217,648 & 133,619 & 7.96 & 217,698 & 134,564 & 7.93 & 0.02 & 0.71 \\
\hline Bolton & 208,193 & 120,790 & 5.61 & 208,085 & 120,350 & 5.62 & -0.05 & -0.36 \\
\hline Pembrokeshire & 93,288 & 51,767 & 5.47 & 93,223 & 51,277 & 5.50 & -0.07 & -0.95 \\
\hline Bury & 144,273 & 86,003 & 5.54 & 144,138 & 85,512 & 5.55 & -0.09 & -0.57 \\
\hline Flintshire & 120,464 & 76,560 & 5.21 & 120,200 & 75,835 & 5.23 & -0.22 & -0.95 \\
\hline Stirling & 69,980 & 42,250 & 6.03 & 69,782 & 41,888 & 6.05 & -0.28 & -0.86 \\
\hline Wrexham & 104,985 & 62,498 & 5.74 & 104,642 & 62,443 & 5.73 & -0.33 & -0.09 \\
\hline Argyll \& Bute & 72,290 & 43,471 & 5.41 & 72,049 & 43,917 & 5.37 & -0.33 & 1.03 \\
\hline South Gloucestershire UA & 199,160 & 136,514 & 5.18 & 198,303 & 136,643 & 5.17 & -0.43 & 0.09 \\
\hline
\end{tabular}


Table A1 continued

Numbers and percentages

\begin{tabular}{|c|c|c|c|c|c|c|c|c|}
\hline \multirow[b]{2}{*}{ Local authority } & \multicolumn{3}{|c|}{ Unreweighted (PWT03) } & \multicolumn{3}{|c|}{ Reweighted (PWT07) } & \multirow{2}{*}{$\begin{array}{r}\text { Difference in } \\
16+ \\
\text { population } \\
\text { (per cent) }\end{array}$} & \multirow{2}{*}{$\begin{array}{r}\text { Difference in } \\
\text { employment } \\
\text { levels } \\
\text { (per cent) }\end{array}$} \\
\hline & $16+$ population & $\begin{array}{r}\text { Employment } \\
\text { levels }\end{array}$ & $\begin{array}{r}\text { Coefficient of } \\
\text { variation }\end{array}$ & $16+$ population & $\begin{array}{r}\text { Employment } \\
\text { levels }\end{array}$ & $\begin{array}{r}\text { Coefficient of } \\
\text { variation }\end{array}$ & & \\
\hline Wiltshire & 350,511 & 226,045 & 5.92 & 348,862 & 224,711 & 5.93 & -0.47 & -0.59 \\
\hline Monmouthshire & 70,196 & 42,797 & 5.50 & 69,856 & 42,128 & 5.53 & -0.48 & -1.56 \\
\hline West Berkshire UA & 119,317 & 81,299 & 5.71 & 118,662 & 80,068 & 5.74 & -0.55 & -1.51 \\
\hline East Sussex & 406,254 & 232,619 & 5.67 & 403,681 & 230,221 & 5.68 & -0.63 & -1.03 \\
\hline Ealing & 242,686 & 151,943 & 7.23 & 240,531 & 149,698 & 7.25 & -0.89 & -1.48 \\
\hline Medway UA & 194,804 & 124,150 & 5.57 & 192,905 & 123,835 & 5.55 & -0.97 & -0.25 \\
\hline Warrington UA & 155,038 & 97,223 & 5.35 & 153,507 & 96,610 & 5.34 & -0.99 & -0.63 \\
\hline Conwy & 90,382 & 47,872 & 5.45 & 89,462 & 47,396 & 5.44 & -1.02 & -0.99 \\
\hline Bromley & 241,939 & 147,726 & 7.57 & 239,111 & 146,168 & 7.57 & -1.17 & -1.05 \\
\hline West Sussex & 621,457 & 374,099 & 4.45 & 613,848 & 368,824 & 4.46 & -1.22 & -1.41 \\
\hline Buckinghamshire & 395,429 & 254,434 & 5.52 & 390,258 & 249,499 & 5.54 & -1.31 & -1.94 \\
\hline Dorset & 334,867 & 191,979 & 5.96 & 330,487 & 185,651 & 6.02 & -1.31 & -3.30 \\
\hline Hillingdon & 201,241 & 122,339 & 7.77 & 198,552 & 118,266 & 7.85 & -1.34 & -3.33 \\
\hline Hampshire & $1,022,070$ & 664,776 & 3.37 & $1,007,801$ & 652,867 & 3.38 & -1.40 & -1.79 \\
\hline Caerphilly & 137,135 & 76,155 & 5.43 & 135,218 & 75,181 & 5.42 & -1.40 & -1.28 \\
\hline Stockport & 227,543 & 145,452 & 5.64 & 224,265 & 143,054 & 5.65 & -1.44 & -1.65 \\
\hline Brighton and Hove UA & 210,967 & 130,570 & 5.28 & 207,705 & 129,207 & 5.27 & -1.55 & -1.04 \\
\hline Hackney & 161,056 & 85,081 & 8.02 & 158,437 & 84,468 & 7.98 & -1.63 & -0.72 \\
\hline Herefordshire, County of UA & 148,175 & 90,014 & 5.42 & 145,532 & 88,855 & 5.40 & -1.78 & -1.29 \\
\hline Harrow & 176,808 & 108,423 & 8.17 & 173,595 & 105,153 & 8.22 & -1.82 & -3.02 \\
\hline Thurrock UA & 117,581 & 73,431 & 5.65 & 115,434 & 72,855 & 5.62 & -1.83 & -0.78 \\
\hline Reading UA & 116,990 & 80,229 & 5.54 & 114,676 & 79,573 & 5.51 & -1.98 & -0.82 \\
\hline Telford and Wrekin & 128,165 & 79,346 & 5.94 & 125,604 & 78,251 & 5.92 & -2.00 & -1.38 \\
\hline East Lothian & 74,877 & 46,862 & 5.32 & 73,351 & 45,444 & 5.35 & -2.04 & -3.03 \\
\hline Croydon & 271,900 & 170,161 & 7.23 & 266,315 & 165,881 & 7.25 & -2.05 & -2.52 \\
\hline Wokingham UA & 123,624 & 84,521 & 5.30 & 121,033 & 82,961 & 5.29 & -2.10 & -1.85 \\
\hline West Lothian & 133,045 & 87,861 & 5.45 & 130,047 & 84,977 & 5.48 & -2.25 & -3.28 \\
\hline Slough UA & 95,390 & 60,921 & 5.33 & 93,055 & 60,027 & 5.30 & -2.45 & -1.47 \\
\hline Barnet & 276,289 & 164,287 & 7.89 & 268,414 & 154,786 & 8.01 & -2.85 & -5.78 \\
\hline East Dunbartonshire & 87,067 & 55,751 & 4.91 & 84,541 & 54,145 & 4.91 & -2.90 & -2.88 \\
\hline Denbighshire & 78,816 & 42,868 & 5.90 & 76,512 & 41,537 & 5.91 & -2.92 & -3.10 \\
\hline Bracknell Forest UA & 90,620 & 63,514 & 5.04 & 87,916 & 61,418 & 5.05 & -2.98 & -3.30 \\
\hline Vale of Glamorgan, The & 100,037 & 58,549 & 5.48 & 97,019 & 56,866 & 5.48 & -3.02 & -2.87 \\
\hline Southend-on-Sea UA & 129,509 & 78,490 & 5.49 & 125,585 & 75,143 & 5.53 & -3.03 & -4.26 \\
\hline Midlothian & 64,627 & 42,414 & 6.14 & 62,644 & 40,296 & 6.20 & -3.07 & -4.99 \\
\hline Bournemouth UA & 133,099 & 78,763 & 5.44 & 128,175 & 76,622 & 5.41 & -3.70 & -2.72 \\
\hline Richmond upon Thames & 149,716 & 98,784 & 7.05 & 143,862 & 94,228 & 7.08 & -3.91 & -4.61 \\
\hline Hammersmith and Fulham & 146,477 & 91,987 & 7.42 & 140,313 & 88,700 & 7.39 & -4.21 & -3.57 \\
\hline Ceredigion & 67,603 & 34,879 & 6.02 & 64,459 & 32,880 & 6.05 & -4.65 & -5.73 \\
\hline East Renfrewshire & 73,785 & 46,135 & 5.52 & 70,292 & 43,393 & 5.55 & -4.73 & -5.94 \\
\hline Poole UA & 113,947 & 68,889 & 5.48 & 108,315 & 64,628 & 5.52 & -4.94 & -6.19 \\
\hline Total & $47,452,934$ & $28,277,079$ & & $48,257,081$ & $28,849,553$ & & 1.69 & 2.02 \\
\hline
\end{tabular}

Note:

1 Key data for UK born and non-UK born population subgroups are available on request. 